\title{
Association between UGT1A1 gene polymorphism and safety and efficacy of irinotecan monotherapy as the third-line treatment for advanced gastric cancer
}

\author{
Toshifumi Yamaguchi ${ }^{1,2,3}$ - Satoru Iwasa ${ }^{3} \cdot$ Hirokazu Shoji $^{3} \cdot$ Yoshitaka Honma $^{3} \cdot$ Atsuo Takashima $^{3} \cdot$ Ken Kato $^{3} \cdot$ \\ Tetsuya Hamaguchi ${ }^{4} \cdot$ Kazuhide Higuchi $^{2} \cdot$ Narikazu Boku $^{3}$
}

Received: 31 August 2018 / Accepted: 17 December 2018 / Published online: 2 January 2019

(c) The International Gastric Cancer Association and The Japanese Gastric Cancer Association 2019

\begin{abstract}
Background While uridine diphosphate glucuronosyltransferase (UGT) 1A1 is a key enzyme in the metabolism of irinotecan, relationship between UGTIAl genotype and safety and efficacy of irinotecan monotherapy in patients with advanced gastric cancer is not clarified.

Methods Efficacy and safety in advanced gastric cancer patients, who were tested for $U G T 1 A I^{*} 6$ and $* 28$ genotype and treated with irinotecan monotherapy as third-line treatment from 2009 to 2014 , were evaluated according to the UGT1AI*6 and $* 28$ genotypes.

Results Among 74 patients of the subjects, the genotypes of UGT1A1 were wild-type (WT) in 37 patients (50\%), single heterozygosity (SH) in $27(36.5 \%)$ and double heterozygosity or homozygosity (Homo/DH) in $10(13.5 \%)$. The initial dose of irinotecan was reduced in 10 patients (27\%) with WT, in $9(33 \%)$ with SH, and in 7 (70\%) with Homo/DH. Median overall survival was 6.9 months, 6.3 months, and 2.8 months in the WT, SH and Homo/DH genotypes, associated with median time to treatment failure of 2.4 months, 2.3 months, and 1.3 months, respectively. Among 36 patients with measurable lesion, disease control rates were $47.6 \%, 41.7 \%$ and 33.3\% in the WT, SH and Homo/DH genotypes. Grade 3 or higher adverse events of special interest were neutropenia (13\%, 22\%, and 64\% for the WT, SH and Homo/DH genotypes), febrile neutropenia (2\%, $7 \%$, and $50 \%$ ) and diarrhea $(6 \%, 5 \%$, and $21 \%)$.

Conclusions The UGTIAl polymorphism may be related to the clinical outcomes of irinotecan monotherapy as the third-line treatment for advanced gastric cancer.
\end{abstract}

Keywords Gastric cancer · UGT1A1 - Irinotecan

Toshifumi Yamaguchi

yamagu.toshifumi@gmail.com

1 Cancer Chemotherapy Center, Osaka Medical College Hospital, 2-7 Daigaku machi, Takatsuki, Osaka 569-8686, Japan

2 Second Department of Internal Medicine, Osaka Medical College, 2-7 Daigaku machi, Takatsuki, Osaka 569-8686, Japan

3 Gastrointestinal Oncology Division, National Cancer Center Hospital, 5-1-1 Tsukiji, Chuo-ku, Tokyo 104-0045, Japan

4 Department of Gastrointestinal Oncology, Saitama Medical University International Medical Center, 1397-1 Yamane, Hidaka, Saitama, Japan

\section{Introduction}

Since combination chemotherapy showed survival benefits compared to best supportive care (BSC) in early 1990s, many phase III trials of first-line chemotherapy including molecular target agents have been conducted. Nowadays, combination chemotherapy with fluoropyrimidine and platinum, with trastuzumab in case of positive HER2, is globally recognized as the standard first-line chemotherapy for advanced gastric cancer [1-3]. In 2010's, survival benefits of monotherapy with irinotecan and docetaxel in the second-line chemotherapy were confirmed in phase III trials [4-6], and irinotecan and weekly paclitaxel showed equivalent efficacy in the WJOG4007 trial [7]. Since superiority of ramucirumab plus weekly paclitaxel over weekly paclitaxel alone was confirmed in the RAINBOW trial, it has 
been recognized as the standard second-line chemotherapy [8], and irinotecan monotherapy has been widely used as the third-line chemotherapy especially in Asian countries. Recently, nivolumab has shown survival benefits compared to best supportive care as the salvage line treatment after at least two prior chemotherapy regimens [9]. Therefore, both nivolumab and irinotecan monotherapy are recommended as the third or later line treatment in the Japanese Gastric Cancer Treatment Guideline.

Irinotecan is hydrolyzed to 7-ethyl-10-hydroxy-camptothecin (SN-38) by carboxylesterases, and this active metabolite SN-38 inhibits topoisomerase I leading to inhibition of DNA replication and transcription $[10,11]$. In clinical practice, however, irinotecan sometimes causes serious adverse events such as neutropenia and delayed-onset diarrhea. Uridine diphosphate glucuronosyltransferase 1A1 (UGT1A1) is a key enzyme in the metabolism and excretion of SN-38 as it transforms SN-38 into glycosylated SN-38 (SN-38G) which is excreted into the bile juice [12-14]. UGT1A1 has genomic polymorphisms such as the UGT1A $1 * 28$ and $* 6$, and activity of UGT1A1 is reduced in individuals with these polymorphisms. Actually, previous studies reported that UGT1AI polymorphisms, especially $U G T I A I * 28$ and $U G T I A I * 6$, is associated with irinotecan-induced severe toxicities in metastatic colorectal cancer patients [15]. Approximately 10\% of the North American population is homozygous for the UGT1A $1 * 28$ allele [16], and patients with homozygous genotype for the $U G T 1 A I^{*} 28$ allele showed an increased risk of neutropenia caused by irinoetecan. Similarly, the UGTIAI*6 variant is frequent in the Asian population (approximately $20 \%$, mainly in East Asia), and metastatic colorectal cancer patients with $U G T I A I^{*} 6$ variants also showed a higher incidence of severe neutropenia $[17,18]$. Therefore, it is recommended that dose reduction of irinotecan should be considered for patients with $U G T I A I * 28$ and/or UGTIAI*6.

However, there are few reports on the associations between $U G T 1 A 1 * 6 / * 28$ polymorphisms and irinotecaninduced toxicities and efficacy in patients with advanced gastric cancer. The purpose of this study is to investigate the relationship between UGTIAI genotype and safety and efficacy of irinotecan alone as third-line chemotherapy for advanced gastric cancer.

\section{Patients and methods}

\section{Patients}

As the source of the subjects in this retrospective analysis, medical records of the 208 patients, who were tested for $U G T 1 A 1 * 6(\mathrm{G} / \mathrm{G}, \mathrm{G} / \mathrm{A}$, and A/A) and $* 28(6 / 6,6 / 7$, and 7/7) genotypes and treated with irinotecan-based chemotherapy for advanced gastric cancer from 2009 to 2014 at
National Cancer Center Hospital, Tokyo, were reviewed. Main selection criteria for this study were as follows: histologically confirmed gastric adenocarcinoma, unresectable or recurrent disease, refractory both to a fluoropyrimidinecontaining first-line chemotherapy, including relapse $<24$ weeks after the final dose of adjuvant chemotherapy with $\mathrm{S}-1$, and to a taxane-containing second-line chemotherapy (paclitaxel, nab-paclitaxel or docetaxel), 20 years or older, Eastern Cooperative Oncology Group (ECOG) performance status (PS) from 0 to 2, sufficient bone marrow, hepatic and renal function, no active concomitant malignancy, and no serious complications.

\section{Treatment}

As a standard regimen, irinotecan at a dose of $150 \mathrm{mg} / \mathrm{m}^{2}$ was administered intravenously every 2 weeks. The treatment was repeated until disease progression, unacceptable toxicity, or patient refusal. Dose modifications of irinotecan, including the initial dose, and treatment delays were decided by the physician's discretion according to the patient general condition, organ function, severity of hematological or nonhematological toxicities, and UGTIAl genotype. In general, the dose was reduced when grade 4 neutropenia, grade 3 or higher febrile neutropenia, and unacceptable grade 2 or higher diarrhea, nausea, and anorexia occurred.

\section{UGT1A1 genotyping}

The genotype of $U G T 1 A 1 * 6$ and $U G T 1 A 1 * 28$ were tested using the Invader® UGT1A1 Molecular Assay kit (Sekisui Medical Co. Ltd, Tokyo, Japan) Genomic DNA for determined to genotype was extracted from mononuclear cells in peripheral blood. genotype was classified to the following three groups: wild-type (WT) with UGT1A $1 * 6 \mathrm{G} / \mathrm{G}$ and $* 28$ $6 / 6$, single heterozygosity $(\mathrm{SH})$ with UGT $1 \mathrm{~A} 1 * 6 \mathrm{G} / \mathrm{G}$ and $* 286 / 7$, or $* 6 \mathrm{G} / \mathrm{A}$ and $* 286 / 6$, and homozygosity/double heterozygosity $(\mathrm{Homo} / \mathrm{DH}$ ) with $* 6 \mathrm{~A} / \mathrm{A}$ and $* 286 / 6$ (Homo for $* 6$ ), $* 6 \mathrm{G} / \mathrm{G}$ and $* 287 / 7$ (Homo for $* 28$ ), or UGT1A1*6 $\mathrm{G} / \mathrm{A}$ and $* 286 / 7$ (DH).

\section{Calculation of relative dose intensity (RDI)}

The dose intensity of irinotecan was calculated by dividing its total actual administered dose by the number of weeks of treatment. The relative dose intensity (RDI) was calculated as a percentage of the dose intensity to the planned target dose $\left(75 \mathrm{mg} / \mathrm{m}^{2}\right)$.

\section{Evaluation and statistical analysis}

The efficacy and safety of third-line irinotecan monotherapy were compared among the three groups classified by the 
genotypes for UGT1A $1 * 28$ or UGT1A $1 * 6$ (wild, SH and Homo/DH). Tumor response was evaluated according to the Response Evaluation Criteria in Solid Tumors (RECIST), version 1.1. Adverse events were assessed according to the Common Terminology Criteria for Adverse Events (CTCAE), version 4.0. Overall survival (OS) was calculated from the date of initiating irinotecan to the date of death from any cause or censored on the last follow-up. Time to treatment failure (TTF) was defined as the interval between the first and last administration of irinotecan. Kaplan-Meier estimates were used to calculate survival probabilities, and the log-rank test was used to compare survival curves. Survival differences between among groups were also evaluated by multivariate analyses using the Cox proportional hazard regression model, and presented as the hazard ratio (HR) and 95\% CI. Multivariate analysis included well-known prognostic factors for advanced gastric cancer such as age $(\leq 65$ versus $>65$ years), gender (male versus female), ECOG PS $(0-1$ versus $\geq 2)$, number of metastatic sites ( 1 versus $\geq 2$ ), histology (intestinal versus diffuse), as well as genotype (WT, SH versus Homo/DH). Statistical analyses were performed using the statistical software 'EZR' (Easy R), which is based on $\mathrm{R}$ and $\mathrm{R}$ commander.

\section{Results}

\section{Patient characteristics}

A total of 208 patients were the source of this retrospective study. Excluding 91 patients receiving combination of chemotherapy containing irinotecan and those receiving irinotecan monotherapy as second- $(n=41)$ or later than 3 rd $(n=2)$ line treatment (Fig. 1). Finally, 74 patients were identified as the subjects of this retrospective study, who were classified into three groups by the genomic types of the UGT1A $* 28$ and $U G T 1 A I^{*} 6$ : WT $(n=37,50 \%), \mathrm{SH}(\mathrm{n}=27,36.5 \%)$, and

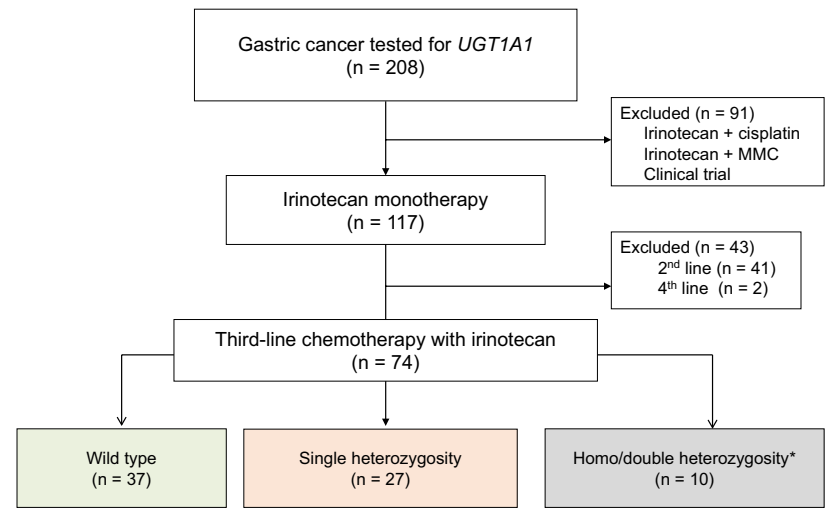

Fig. 1 CONSORT diagram of gastric cancer patients tested for the UGTA1 genotype
Homo/DH $(n=10,13.5 \%)$. Their baseline characteristics are shown in Table 1.

\section{Initial dose and drug delivery of irinotecan}

The initial dose of irinotecan was reduced in $27 \%(10 / 37)$ of the patients in the WT group $\left(120 \mathrm{mg} / \mathrm{m}^{2}, 6\right.$ patients; $100 \mathrm{mg} / \mathrm{m}^{2}, 4$ patients), in $33 \%(9 / 27)$ of those in the $\mathrm{SH}$ group $\left(120 \mathrm{mg} / \mathrm{m}^{2}, 8\right.$ patients; $100 \mathrm{mg} / \mathrm{m}^{2}, 1$ patient $)$, and in $70 \%(7 / 10)$ of those in the Homo/DH group $(120 \mathrm{mg} /$ $\mathrm{m}^{2}, 3$ patients; $100 \mathrm{mg} / \mathrm{m}^{2}, 4$ patients). In the total 26 patients whose initial dose was reduced, six patients (23\%) required further dose reduction due to adverse events. The relative dose intensity of irinotecan was lower in the Homo/DH group (52.5\%) than in the WT (77.6\%) and SH groups $(70.3 \%)$. The median number of administration of irinotecan was smaller in the Homo/DH group ( 2 cycles, range: 1-6 cycles) than in the WT (4 cycles, range: $1-34$ cycle) and SH groups (3 cycles, range: $1-22$ cycles). With a median follow-up of 7.2 months, the median time to

Table 1 Patients' characteristics in third-line chemotherapy

\begin{tabular}{|c|c|c|c|}
\hline & $\begin{array}{l}\text { Wild-type } \\
(n=37)\end{array}$ & $\begin{array}{l}\text { Single } \\
\text { heterozygous } \\
(n=27)\end{array}$ & $\begin{array}{l}\text { Double } \\
\text { heterozygous/ } \\
\text { Homozygous } \\
(n=10)\end{array}$ \\
\hline Age (median) & $65(41-80)$ & $67(36-77)$ & $67(47-74)$ \\
\hline \multicolumn{4}{|l|}{ Gender } \\
\hline Male & 27 & 20 & 4 \\
\hline Female & 10 & 7 & 6 \\
\hline \multicolumn{4}{|l|}{ ECOG PS } \\
\hline 0 & 3 & 2 & 1 \\
\hline 1 & 21 & 23 & 8 \\
\hline 2 & 13 & 2 & 1 \\
\hline \multicolumn{4}{|l|}{ Histological type } \\
\hline Intestinal & 18 & 8 & 3 \\
\hline Diffuse & 19 & 19 & 7 \\
\hline \multicolumn{4}{|c|}{ Number of metastatic sites } \\
\hline 1 & 19 & 14 & 4 \\
\hline$\geq 2$ & 18 & 13 & 6 \\
\hline \multicolumn{4}{|l|}{ Target lesion } \\
\hline Yes & 27 & 18 & 3 \\
\hline \multicolumn{4}{|l|}{ Site of metastasis } \\
\hline Lymph node & 20 & 12 & 7 \\
\hline Liver & 16 & 8 & 3 \\
\hline Peritoneum & 18 & 15 & 6 \\
\hline \multicolumn{4}{|c|}{ Previous therapies } \\
\hline $\begin{array}{l}\text { Fluoropyrimi- } \\
\text { dine }\end{array}$ & 37 & 27 & 10 \\
\hline Platinum & 34 & 24 & 8 \\
\hline Taxane & 37 & 27 & 10 \\
\hline
\end{tabular}

ECOG PS Eastern Cooperative Oncology Group Performance Status 


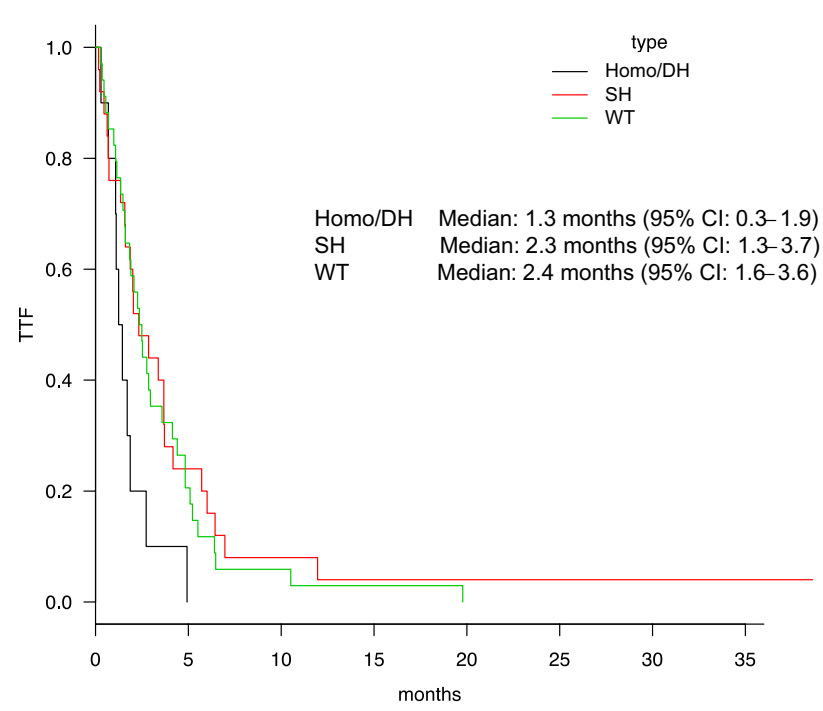

Fig. 2 Time to treatment failure of third-line irinotecan monotherapy in the WT, $\mathrm{SH}$, and Homo/DH groups

treatment failure (mTTF) was 2.4 months (95\% CI 1.6-3.6) in the WT group, 2.3 months (95\% CI 1.3-3.7) in the SH group, and 1.3 months (95\% CI 0.3-1.9) in the Homo/DH group (Fig. 2). Reasons for treatment discontinuation were disease progression (78.3\%), hematological adverse events (2.7\%) and non- hematological adverse events $(5.4 \%)$ and others $(13.6 \%)$ in the WT group, and those were $59.2 \%$, $7.4 \%, 7.4 \%$ and $26.0 \%$ in the SH group, $30.0 \%, 40.0 \%$, $10.0 \%$ and $20.0 \%$ in the Homo/DH group, respectively.

\section{Toxicity of irinotecan in third-line chemotherapy}

The hematological and non-hematological adverse events observed during the irinotecan monotherapy are listed in Table 3. The incidences of grade 3 or 4 neutropenia (50\%) and febrile neutropenia (40\%) in the Homo/DH group were significantly higher than in the other groups. The incidences of grade 3 or 4 diarrhea in the WT, SH, and Homo/DH groups were $8.1 \%, 3.7 \%$, and $10 \%$, respectively. No treatment-related deaths were observed in any group (Table 2).
Table 3 Response in measurable lesions

\begin{tabular}{lllll}
\hline & Total & WT & SH & Homo/DH \\
\hline PR & $2(5.5 \%)$ & $1(4.8 \%)$ & $1(8.3 \%)$ & 0 \\
SD & $14(39.0 \%)$ & $9(42.8 \%)$ & $4(33.4 \%)$ & $1(33.3 \%)$ \\
PD & $20(55.5 \%)$ & $11(52.4 \%)$ & $7(58.3 \%)$ & $2(66.6 \%)$ \\
NE & 7 & 2 & 3 & 2 \\
ORR (\%) & 5.5 & 4.8 & 8.3 & 0 \\
DCR $(\%)$ & 44.5 & 47.6 & 41.6 & 33.3 \\
\hline
\end{tabular}

$P R$ partial response, $S D$ stable disease, $P D$ progressive disease, $N E$ not evaluate, $O R R$ overall response rate, $D C R$ disease control rate

\section{Efficacy of irinotecan as third-line treatment}

Among the 36 patients with measurable lesion at baseline, 2 achieved a partial response (5.5\%), 14 showed stable disease (39\%), and 20 showed progression disease (55.5\%), resulting in a response rate of $5.5 \%$, and disease control rate of $44.5 \%$. Disease control rate was $47.6 \%$ in the WT group, $41.7 \%$ in the $\mathrm{SH}$ group, and $33.3 \%$ in the Homo/DH group, respectively (Table 3 ). Median overall survival was 4.6 months (95\% CI 3.4-6.2) in all patients. Median overall survival was 6.9 months (95\% CI 3.9-7.9) in the WT group, 6.3 months (95\% CI 3.3-8.3) in the SH group, and 2.8 months (95\% CI 1.2-3.4) in the Homo/DH group, respectively (Fig. 3).

\section{Prognostic factors}

Univariate and multivariate analyses for prognostic factors of OS are shown in Table 4. Risk factors for poor OS were the UGTIAl genotype $(p=0.034)$ in univariate analysis and ECOG PS $2(p<0.0001)$ in univariate and multivariate analysis.

\section{Discussion}

In summary, this study showed association between the UGTIAl genotype and clinical outcomes such as disease control, time to treatment failure, overall survival, and severe adverse events in Japanese patients with advanced gastric cancer who received irinotecan monotherapy in the thirdline setting.
Table 2 Adverse events of special interest

\begin{tabular}{|c|c|c|c|c|c|c|}
\hline & \multicolumn{2}{|c|}{$\mathrm{WT} n(\%)$} & \multicolumn{2}{|l|}{$\mathrm{SH} n(\%)$} & \multicolumn{2}{|c|}{ Homo/DH $n(\%)$} \\
\hline & All & Gr3 & All & $\mathrm{Gr}$ & All & Gr3 \\
\hline Leukopenia & $23(67)$ & $7(19)$ & $16(59)$ & $4(14.8)$ & $8(80)$ & $5(50)$ \\
\hline Neutropenia & $19(56)$ & $5(14)$ & $14(52)$ & $4(14.8)$ & $8(80)$ & $5(50)$ \\
\hline Febrile neutropenia & $1(2.7)$ & $1(2.7)$ & $1(3.7)$ & $1(3.7)$ & $4(40)$ & $4(40)$ \\
\hline Diarrhea & $6(16.2)$ & $3(8.1)$ & $4(14.8)$ & $1(3.7)$ & $1(10)$ & $1(10)$ \\
\hline
\end{tabular}




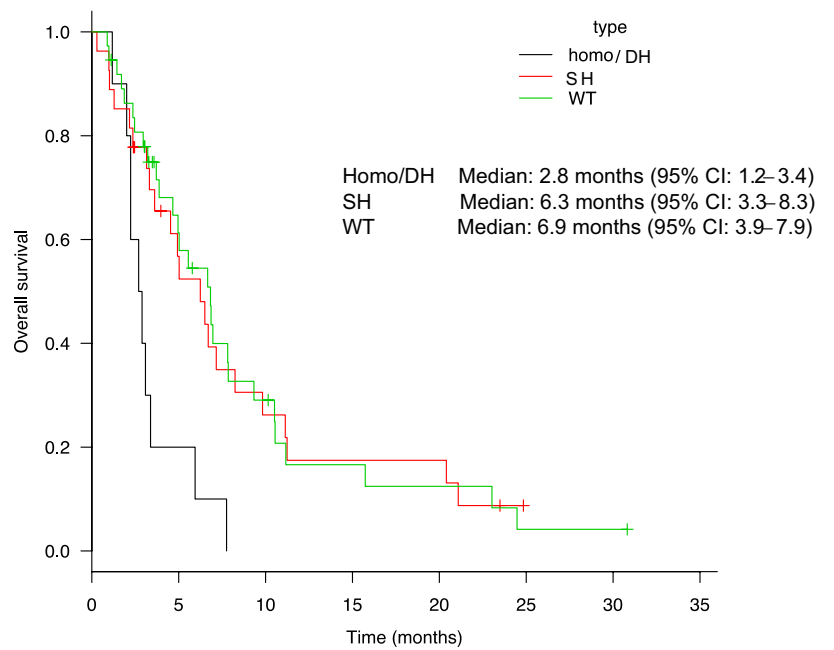

Fig. 3 Kaplan-Meier analysis of overall survival (third-line chemotherapy) in the WT, $\mathrm{SH}$, and Homo/DH group

As for toxicity, irinotecan monotherapy was well tolerated in the WT and SH groups. In contrast to the results that there were no differences in incidences of any toxicities between the WT and SH groups, the incidences of grade 3 or 4 adverse events in the Homo/DH group were higher than those in the WT and SH groups, especially leucopenia, febrile neutropenia, and diarrhea. The homozygous genotype was associated with a twofold higher risk of severe neutropenia compared with the WT and SH groups, despite using a lower starting dose in the Homo/DH group. These results were consistent to previous similar study of patients with advanced colorectal cancer. Ichikawa et al. evaluated the effects of UGT1A1 genotypes and non-genetic factors on the efficacy and safety of irinotecan-based regimens in
1312 patients with advanced colorectal cancer [19]. As for toxicities, the homozygous genotype was associated with a twofold higher risk of severe neutropenia in the first course as compared with wild-type, despite using a lower starting dose in the homozygous group. In this study, although $70 \%$ of patients in the Homo/DH group received initially reduced dose of irinotecan, they experienced severe adverse events. Therefore, it seems difficult to use the standard dose of irinotecan for patients with UGTIAl Homo/DH polymorphism.

Furthermore, this study showed that survival benefits of third-line irinotecan was lower in patients with the Homo/ DH genotype UGT1Al polymorphism compared with patients with the WT and SH polymorphisms (OS: 2.8 versus 6.9 and 6.3 months, respectively). The median OS in the WT and SH groups was similar to those reported in previous retrospective studies of third- or later-line treatment with irinotecan ranging 4.0-6.6 months [20-22]. However, the median OS in the Homo/DH group seemed as short as those of best supportive care in pivotal phase III studies $(2.4,3.6$, $3.8,4.1$, and 4.3 months in AIO study [4], COUGAR-02 study [5], Korean study [6], ONO-4538-12 study [9], and GRANITE-1 [23] study, respectively). Although there were no remarkable differences in disease control rates among the three groups, time to treatment failure is shorter in the Homo/DH group than others, and there are more patients whose treatment was discontinued due to adverse events. It is considered that serious adverse events which caused short TTP and deteriorated the patient's condition might cause lack of benefits of irinotecan for the patients in the Homo/ DH group.

Recently, nivolumab has been shown to provide a significant survival benefit in patients with refractory to standard treatment (ONO-4538-12 study), and the Ministry of
Table 4 Univariate and multivariate analysis for overall survival

\begin{tabular}{|c|c|c|c|c|}
\hline \multirow[t]{2}{*}{ Covariate } & \multicolumn{2}{|l|}{ Univariate analysis } & \multicolumn{2}{|l|}{ Multivariate analysis } \\
\hline & $\mathrm{HR}(95 \% \mathrm{CI})$ & $p$ value & $\mathrm{HR}(95 \% \mathrm{CI})$ & $p$ value \\
\hline $\begin{array}{l}\text { ECOG PS } \\
2 \text { versus } 0-1\end{array}$ & $6.027(2.712-13.394)$ & $<0.0001$ & $7.135(2.867-17.756)$ & $<0.0001$ \\
\hline $\begin{array}{l}\text { Age } \\
\geq 65 \text { vs. }<65\end{array}$ & $1.008(0.598-1.698)$ & 0.976 & $1.283(0.726-2.266)$ & 0.39 \\
\hline $\begin{array}{l}\text { Gender } \\
\text { Female versus male }\end{array}$ & $1.170(0.657-2.081)$ & 0.594 & $1.306(0.684-2.492)$ & 0.419 \\
\hline $\begin{array}{l}\text { No. of metastatic sites } \\
\geq 2 \text { versus } 1\end{array}$ & $1.611(0.965-2.691)$ & 0.068 & $1.335(0.741-2.405)$ & 0.335 \\
\hline $\begin{array}{l}\text { Histology } \\
\text { Diffuse versus intestinal }\end{array}$ & $1.269(0.763-2.113)$ & 0.359 & $1.403(0.811-2.426)$ & 0.226 \\
\hline $\begin{array}{l}\text { UGT1A1 genotype } \\
\text { SH, Homo/DH versus WT }\end{array}$ & $1.525(1.033-2.251)$ & 0.034 & $1.306(0.684-2.492)$ & 0.087 \\
\hline
\end{tabular}

$H R$ hazard ratio, CI confidence interval, ECOG PS Eastern Cooperative Oncology Group Performance Status

*Right-hand sides were used as reference groups 
Health Labour and Welfare of Japan approved nivolumab for advanced gastric cancer as salvage line treatment in 2017. In the Gastric Cancer Treatment Guide Lines 2018 published by the Japanese Gastric Cancer Association, both irinotecan and nivolumab are recommended in the third or later line treatment. Among patients assigned to best supportive care were enrolled in the ONO-4538-12 study, 25\% of them did not receive irinotecan as prior treatment and only $5.5 \%$ of them received irinotecan after placebo. Therefore, precisely, it is still unclear whether irinotecan or nivolumab should be administered first if UGTIAl polymorphism is not taken into account. However, together with the efficacy and toxicities of patients with the UGTIAl Homo/DH polymorphism in this study, genotype testing of UGTIAl is recommended for selecting the third-line therapy either irinotecan or nivolumab. And it is suggested that irinotecan monotherapy as the third-line therapy may not be selected preferentially than nivolumab for advanced gastric cancer patients with the UGTIAl Homo/DH polymorphism.

The current study has some limitations. This is a retrospective study which could not collect the precise data of adverse events and quality of life, no data of pharmacokinetics, and there were no pre-specified criteria for dose reduction, rest and discontinuation of irinotecan. The small sample size at single-center, especially, including only 10 UGT1A1 Homo/DH patients, could not adjust the difference in patient's background even by multivariate analysis. These limitations might make some bias in this study.

In conclusion, since Homo/DH patients showed unfavorable clinical outcomes associated with a high risk of grade 3 or higher adverse events, irinotecan may not be administered as third-line or later-line therapy in advanced gastric cancer patients with the UGTIAI Homo/DH polymorphism.

\section{Compliance with ethical standards}

Conflict of interest None of the authors have conflicts of interest to declare.

Research involving human participants All procedures followed were in accordance with the ethical standards of the responsible committee on human experimentation (institutional and national) and with the Helsinki Declaration of 1964 and later versions. Informed consent or substitute for it was obtained from all patients for being included in the study.

\section{References}

1. Koizumi W, Narahara H, Hara T, Takagane A, Akiya T, Takagi M, et al. Randomized phase III study of S-1 alone versus S-1+ cisplatin in the treatment for advanced gastric cancer (The SPIRITS trial) SPIRITS:S-1 plus cisplatin vs S-1 in RCT in the treatment for stomach cancer. Lancet Oncol. 2008;9:215-21.

2. Boku N, Yamamoto S, Shirao K, Doi T, Sawaki A, Koizumi W, et al. Fluorouracil versus combination of irinotecan plus cisplatin versus $\mathrm{S}-1$ in metastatic gastric cancer:a randomized phase 3 study. Lancet Oncol. 2009; 10:1063-9.

3. Bang YJ, Van Cutsem E, Feyereislova A, Chung HC, Shen L, Sawaki A, et al. Trastuzumab in combination with chemotherapy versus chemotherapy alone for treatment of HER2-positive advanced gastric or gastro-oesophageal junction cancer (ToGA):a phase 3, open-label, randomised controlled trial. Lancet. 2010;376:687-97.

4. Thuss-Patience PC, Kretzschmar A, Bichev D, Deist T, Hinke A, Breithaupt K, et al. Survival advantage for irinotecan versus best supportive care as second-line chemotherapy in gastric cancer-a randomised phase III study of the Arbeitsgemeinschaft Internistische Onkologie (AIO). Eur J Cancer. 2011 Oct;47(15):2306-14.

5. Ford HE, Marshall A, Bridgewater JA, Janowitz T, Coxon FY, Wadsley J, et al. Docetaxel versus active symptom control for refractory oesophagogastric adenocarcinoma (COUGAR-02): an open-label, phase 3 randomised controlled trial. Lancet Oncol. 2014 15(1):78-86.

6. Kang JH, Lee SI, Lim DH, Park KW, Oh SY, Kwon HC, et al. Salvage chemotherapy for pretreated gastric cancer: a randomized phase III trial comparing chemotherapy plus best supportive care with best supportive care alone. J Clin Oncol. 2012 30(13):1513-8.

7. Hironaka S, Ueda S, Yasui H, Nishina T, Tsuda M, Tsumura T, et al. Randomized, open-label, phase III study comparing irinotecan with paclitaxel in patients with advanced gastric cancer without severe peritoneal metastasis after failure of prior combination chemotherapy using fluoropyrimidine plus platinum: WJOG 4007 trial. J Clin Oncol. 2013 31(35):4438-44.

8. Wilke H, Muro K, Van Cutsem E, Oh SC, Bodoky G, Shimada $\mathrm{Y}$, et al. Ramucirumab plus paclitaxel versus placebo plus paclitaxel in patients with previously treated advanced gastric or gastro-oesophageal junction adenocarcinoma (RAINBOW): a double-blind, randomised phase 3 trial. Lancet Oncol. 2014;15(11):1224-35.

9. Kang YK, Boku N, Satoh T, Ryu MH, Chao Y, Kato K, et al. Nivolumab in patients with advanced gastric or gastro-oesophageal junction cancer refractory to, or intolerant of, at least two previous chemotherapy regimens (ONO-4538-12, ATTRAC TION-2): a randomised, double-blind, placebo-controlled, phase 3 trial. Lancet. 2017 390(10111):2461-71.

10. Fujita K, Sparreboom A. Pharmacogenetics of irinotecan disposition and toxicity: a review. Curr Clin Pharmacol. 2010 5(3):209-17.

11. O'Dwyer PJ, Catalano RB. Uridine diphosphate glucuronosyltransferase (UGT) $1 \mathrm{~A} 1$ and irinotecan: practical pharmacogenomics arrives in cancer therapy. J Clin Oncol. 2006 24(28):4534-8.

12. Fujita K, Ando Y, Nagashima F, Yamamoto W, Yamamoto W, Eodo H, Araki K, et al. Genetic linkage of UGT1A7 and UGT1A9 polymorphisms to UGT1A $1 * 6$ is associated with reduced activity for SN-38 in Japanese patients with cancer. Cancer Chemother Pharmacol. 2007;60:515-22.

13. Ando Y, Saka H, Ando M, Sawa T, Muro K, Ueoka H, et al. Polymorphisms of UDP-glucuronosyltransferase gene and irinotecan toxicity: a pharmacogenetic analysis. Cancer Res. 2000 60(24):6921-6.

14. Innocenti F, Undevia SD, Iyer L, Chen PX, Das S, Kocherginsky $\mathrm{M}$, et al. Genetic variants in the UDP-glucuronosyltransferase 1A1 gene predict the risk of severe neutropenia of irinotecan. J Clin Oncol. 2004 22(8):1382-8.

15. D'Andrea M, Pasetto LM, Pessa S, Russo A, Buonadonna A, D'Andrea M, et al. The role of UGT1A1*28 polymorphism in the 
pharmacodynamics and pharmacokinetics of irinotecan in patients with metastatic colorectal cancer. J Clin Oncol. 2006;24:3061-8.

16. Kurose K, Sugiyama E, Saito Y. Population differences in major functional polymorphisms of pharmacokinetics/pharmacodynamics-related genes in Eastern Asians and Europeans: implications in the clinical trials for novel drug development. Drug Metab Pharmacokinet. 2012;27(1):9-54.

17. Onoue M, Terada T, Kobayashi M, Katsura T, Matsumoto S, Yanagihara K, et al. UGT1A1*6 polymorphism is most predictive of severe neutropenia induced by irinotecan in Japanese cancer patients. Int J Clin Oncol. 2009 14(2):136-42.

18. Xu C, Tang X, Qu Y, Keyoumu S, Zhou N, Tang Y.et al. UGT1A1 gene polymorphism is associated with toxicity and clinical efficacy of irinotecan-based chemotherapy in patients with advanced colorectal cancer. Cancer Chemother Pharmacol. 2016 78(1):119-30.

19. Ichikawa W, Uehara K, Minamimura K, Tanaka C, Takii Y, Miyauchi $\mathrm{H}$, et al. An internally and externally validated nomogram for predicting the risk of irinotecan-induced severe neutropenia in advanced colorectal cancer patients. Br J Cancer. 2015 112(10):1709-16.

20. Makiyama A, Arimizu K, Hirano G, Makiyama C, Matsushita Y, Shirakawa T, et al. Irinotecan monotherapy as third-line or later treatment in advanced gastric cancer. Gastric Cancer. 2018 21(3):464-72.

21. Kawakami T, Machida N, Yasui H, Kawahira M, Kawai S, Kito Y, et al. Efficacy and safety of irinotecan monotherapy as third-line treatment for advanced gastric cancer. Cancer Chemother Pharmacol. 2016 78(4):809-14.

22. Nishimura T, Iwasa S, Nagashima K, Okita N, Takashima A, Honma Y, et al. Irinotecan monotherapy as third-line treatment for advanced gastric cancer refractory to fluoropyrimidines, platinum, and taxanes. Gastric Cancer. 2017 20(4):655-62.

23. Ohtsu A, Ajani JA, Bai YX, Bang YJ, Chung HC, Pan HM, et al. Everolimus for previously treated advanced gastric cancer: results of the randomized, double-blind, phase III GRANITE-1 study. J Clin Oncol. 2013 31(31):3935-43.

Publisher's Note Springer Nature remains neutral with regard to jurisdictional claims in published maps and institutional affiliations. 